\title{
Tuberculous broncho-oesophageal fistula: images demonstrating the pathogenesis
}

\author{
Pierre Goussard • Savvas Andronikou
}

Received: 5 October 2009/Revised: 24 November 2009/Accepted: 28 November 2009 / Published online: 9 February 2010

(C) Springer-Verlag 2010

An 18-month-old girl with known pulmonary tuberculosis, on treatment for 5 months, presented with a history of coughing after feeding and recurrent infections of the left lower lobe. Chest radiograph showed a large right-side mediastinal lymph node, patent airways and pneumonic changes in the right middle lobe. There was gaseous distension of the stomach. Flexible bronchoscopy confirmed a suspected bronchooesophageal fistula (BOF) in the left main bronchus with caseating material eroding into the lumen. Gastroscopy visualised a calcified lesion in the oesophagus.

Barium swallow (Fig. 1) confirmed the left-side BOF with contrast medium demonstrated in the tracheo-bronchial tree

Fig. 1 Upper gastrointestinal contrast study

P. Goussard $(\bowtie)$

Department of Paediatrics and Child Health,

University of Stellenbosch, Tygerberg Children's Hospital,

P.O. Box 19063, Tygerberg 7505, South Africa

e-mail: pgouss@sun.ac.za

S. Andronikou

Department of Radiology, University of Cape Town,

Cape Town, South Africa

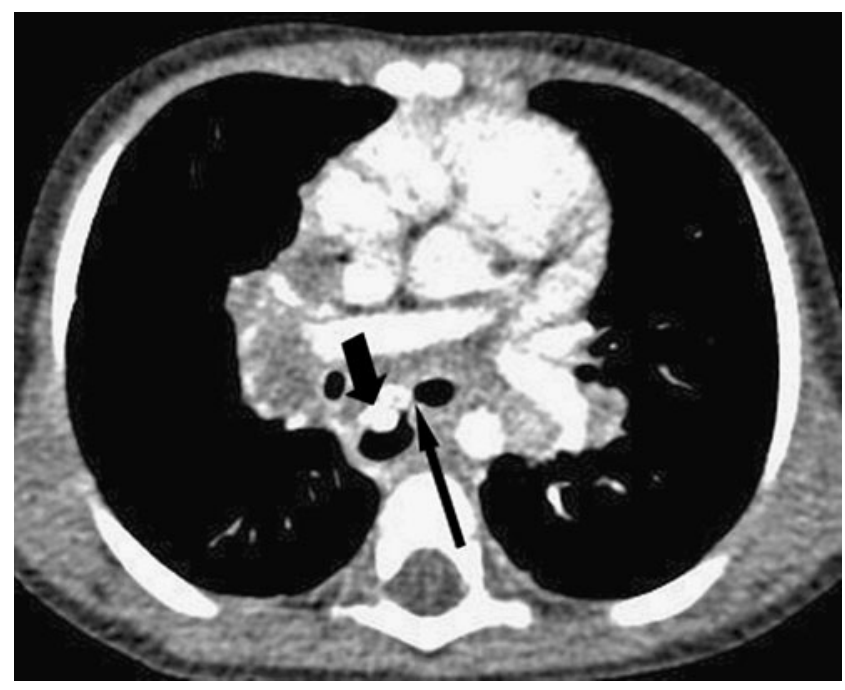

Fig. 2 Axial contrast-enhanced CT scan of the chest

during the 'swallow' phase (arrow), without evidence of palatopharyngeal aspiration. Contrast-enhanced CT scan of the chest (Fig. 2) showed enlarged, calcified subcarinal lymphadenopathy compressing the left main bronchus and bronchus intermedius with erosion into the oesophagus (short arrow). A fistula was demonstrated between the oesophagus and the left main bronchus (long arrow). There was bilateral hilar lymphadenopathy. A calcified lymph node was seen extending from the oesophagus to the left main bronchus in the position of the BOF.

These images confirm that BOF secondary to tuberculosis in children is most likely caused by glandular erosion of a lymph node into both the oesophagus and bronchus, although other explanations for BOF have been reported in adults $[1,2]$.

\section{References}

1. Gie RP, Goussard P, Kling S et al (2004) Unusual forms of intrathoracic tuberculosis in children and their management. Paediatr Respir Rev 5(Suppl A):S139-141

2. Mahapatro S, Kane D, Dave S et al (2007) Post-tuberculous bronchoesophageal fistula. Ind J Thorac Cardiovasc Surg 23:161-163 